\title{
Eops: ¿La dermatoscopía de epiluminiscencia es superior a la inspección clínica en la detección temprana del melanoma?
}

\begin{abstract}
Resumen
En la presente viñeta se presenta un paciente con antecedentes familiares de melanoma. Dentro de las posibles estrategias de rastreo en esta población, el médico tratante se pregunta acerca de la dermatoscopía de epiluminiscencia, también conocida como DIAR-d. Se realiza una pregunta que generó el caso, y se diseña una estrategia de búsqueda. Luego se introduce brevemente el problema del melanoma, se resume los dos artículos más relevantes detectados, que concluyen que adicionar la dermatoscopía, realizada por expertos, al diagnóstico clínico de lesiones sospechosas logra disminuir los diagnósticos falsos positivos, mejorar el valor predictivo positivo, y reducir las biopsias innecesarias. Sin embargo, estos datos deben ser interpretados con cautela: actualmente la fuerza de tareas preventiva de EE.UU no recomienda el rastreo de melanoma en esta población.
\end{abstract}

Villalón G. ¿La dermatoscopía de epiluminiscencia es superior a la inspección clínica en la detección temprana del melanoma? Evid. actual. práct. ambul; 9(1): 16-17. Ene-Feb 2006

\section{Escenario Clínico}

Un médico de familia atiende a un paciente de 40 años cuyo padre falleció de melanoma, por lo cual realiza control periódico de sus lunares. El paciente consulta para conocer la opinión de su médico acerca de un nuevo método de estudio de lunares del cual le llegó un folleto a su domicilio (dermatoscopía de epiluminiscencia o DIAR-d). El médico se pregunta sobre la utilidad de esta nueva tecnología disponible.

\section{Pregunta que generó el caso}

¿En pacientes con riesgo aumentado para desarrollar melanoma (población), el rastreo de estas lesiones usando la dermatoscopía de epiluminiscencia (test diagnóstico/intervención) comparada con la inspección clínica convencional (comparación), aumenta la detección de lesiones tempranas y reduce la mortalidad por melanoma (resultados)?

\section{El problema}

La incidencia de melanoma ha ido en aumento a lo largo de las últimas décadas en todo el mundo, siendo el riesgo actual de desarrollarlo en algún momento de la vida de alrededor de 1,74\% y $1,28 \%$ en varones y mujeres caucásicos respectivamente. Ciertos factores se hallan asociados a un mayor riesgo de melanoma, entre ellos el color claro de piel y cabello (Odds Ratio* [OR] 1,4-3,5), gran número de lesiones lentiginosas actínicas (OR 3,5), frecuente exposición solar (OR 2,63), crecimiento de un nevo (OR 2,3), piel que no se broncea fácilmente (OR 1,98), e historia familiar de melanoma (OR 1,8)

La enfermedad se comporta de manera silente como una lesión pigmentada que modifica progresivamente sus características, pero que muchas veces pasa desapercibida hasta que tiene compromiso metastático. En el $50 \%$ de los casos se origina a partir de piel sana. La forma clínica más común está representada por la variedad de diseminación superficial $(70 \%)$ que presenta una fase inicial de crecimiento radial de duración variable (aproximadamente cinco años) previo a su crecimiento en profundidad y consecuente diseminación sistémica (linfohemática, cerebro, hígado, pulmón).

Clínicamente las lesiones pigmentadas pueden ser evaluadas a través de la regla del ABCDE (Asimetría de la lesión, Bordes irregulares, Color variado, Diámetro mayor a $6 \mathrm{~mm}$ y Evolución de la lesión en el tiempo, generalmente por aumento de tamaño), o por reglas que tienen en cuenta además el sangrado de las lesiones, síntomas sensitivos e inflamación local.

Recientemente se ha incorporado a la estrategia de evaluación clínica el uso de la dermatoscopía, que consiste en un dispositivo manual de amplificación de las lesiones (x10 o mayor) que analiza las mismas a través de diferentes escalas que consideran fundamentalmente la arquitectura de la lesión y su evolución en el tiempo. ${ }^{1,2}$

\section{Estrategia de búsqueda}

Se realizó una búsqueda en Medline utilizando los filtros metodológicos (Clinical Queries) para estudios de diagnóstico tomando como palabras clave los términos MeSH "dermoscopy melanoma mass screening", con lo cual se obtuvieron 38 citas, de las cuales dos resultaron atractivas para contestar la pregunta.

\section{Resumen de la evidencia}

Fuente 1: Bafounta ML, Beauchet A, Aegerter P. Is dermoscopy (epiluminescence microscopy) useful for the diagnosis of melanoma?: results of a meta-analysis using techniques adapted to the evaluation of diagnostic test. Archives of dermatology 2001;137(10):1343-1350.

Objetivo: Evaluar la precisión de la dermatoscopía vs. la inspección clínica realizada por observadores experimentados en el diagnóstico de melanoma.

Fuente de datos: Medline, EMBASE, PASCAL-BIOMED (reporte de congresos), BIUM (Biblioteca médica inter-universitaria, París) hasta mayo de 2000.

Selección de estudios y extracción de datos: Se seleccionaron estudios de corte transversal que compararan de manera ciega la performance diagnóstica de la dermatoscopía (complejidad instrumental variable) contra la inspección clínica para el diagnóstico de melanoma, utilizando la histopatología como test de referencia o Gold Standard*.

Los estudios debían proveer datos de interés como el ámbito de atención, número total y espectro de lesiones estudiadas, característica del instrumento dermatoscópico y criterios diagnósticos utilizados, así como todos los datos necesarios para calcular los valores de sensibilidad ${ }^{*}$, especificidad ${ }^{*}$, y coeficientes de probabilidad o likelihood ratios (LR)*. Finalmente se incluyeron ocho estudios para realizar el metanálisis.

Resultados principales: Todos los estudios fueron realizados en centros dermatológicos de derivación, donde se utilizaron instrumentos dermatoscópicos de complejidad variable. La incidencia de melanoma varió entre 3 al $49 \%$ de las lesiones. Los resultados de los estudios individuales pueden compararse en la tabla 1.

Utilizando los valores operativos individuales se construyo una curva $\mathrm{ROC}^{*}$ para comparar gráficamente ambas estrategias. El análisis en conjunto de los datos reveló una capacidad diagnóstica para la dermatoscopía e inspección clínica, expresada en LR+, de 9 (IC95\% 5,6-19) vs. 3,7 (IC95\% 2,8-5,3); y LR- de 0,11 (IC95\% $0,05-0,18)$ y 0,27 (IC95\% 0,19-0,36) respectivamente. La dermatoscopía mostró asimismo mayor poder discriminatorio con OR estimados de 76 (IC95\% 25-223) vs. 16 (IC95\% 9-31) para la inspección clínica. 
Tabla 1: Características operativas de los tests en los diferentes estudios.

\begin{tabular}{|c|c|c|c|c|c|c|c|c|c|c|c|c|}
\hline \multirow{3}{*}{$\begin{array}{l}\text { Fuente } \\
\text { Benelli }\end{array}$} & \multicolumn{6}{|c|}{ Diannóstico Clińco } & \multicolumn{6}{|c|}{ Dematoseopia } \\
\hline & $\mathbf{s}$ & $\bar{E}$ & VPP & VFIII & Iit & Fis- & s & E & VPP & IVPI & Lit & $\mathrm{IR}-$ \\
\hline & 0.85 & 0.55 & 25 & 95,4 & 1,89 & 0,27 & 0,80 & 0,89 & 55,8 & 96,2 & 7,18 & 0,23 \\
\hline Carli & 0,50 & 0,82 & 50 & 81,8 & 2,75 & 0,61 & 0,75 & 0,91 & 75 & 90,9 & 8,25 & 0,28 \\
\hline Gristofolini & 0,85 & 0,75 & 37,3 & 97 & 3,38 & 0,2 & 0,65 & 0,79 & 35,4 & 92,8 & 3,12 & 0,44 \\
\hline Oumner & 0,65 & 0,93 & 21 & 98,9 & 9,32 & 0,38 & 0,96 & 0,98 & 58 & 99,9 & 47,89 & 0,04 \\
\hline Krahn & 0,79 & 0,78 & 77,5 & 80 & 3,62 & 0,26 & 0,90 & 0,93 & 92,1 & 90,5 & 12,26 & 0,11 \\
\hline Lorentiven & 0,78 & 0,89 & 65,6 & 93,7 & 7,1 & 0,25 & 0,52 & 0,94 & 69,9 & 88 & 8,66 & 0,51 \\
\hline Sover & 0,94 & 0,82 & 78,2 & 95,1 & 5,19 & 0,08 & 0,94 & 0,82 & 78,2 & 95,1 & 5,19 & 0,08 \\
\hline Stanganelli & 0,67 & 0,84 & 53 & 90,6 & 4,22 & 0,39 & 0,93 & 0,94 & 79,7 & 98 & 14,77 & 0,08 \\
\hline
\end{tabular}

S: sensibilidad; E: especificidad; VPP: Valor Predictivo Positivo; VPN: Valor Predictivo Negativo; LR: coeficiente de probabilidad positivo y negativo (ver definiciones en el glosario).

Conclusiones: en usuarios experimentados, la dermatoscopía es más precisa que la inspección clínica para el diagnóstico de melanoma en lesiones pigmentadas.

Fuente 2: Carli P, Mannone F, De Giorgi V. The problem of falsepositive diagnosis in melanoma screening: the impact of dermoscopy. Melanoma Research 2003;13:179-182.

Objetivo: Evaluar el impacto de la dermatoscopía de epiluminiscencia en la reducción de resultados falsos positivos de la inspección clínica.

Diseño, lugar y pacientes: estudio observacional prospectivo, realizado en un centro dermatológico de Florencia, Italia. Los pacientes eran derivados por sus médicos de atención primaria tanto por lesiones pigmentadas sospechosas, o por factores de riesgo aumentados para melanoma.

Descripción del test y Test de Referencia*: Se inspeccionaban todas las lesiones pigmentadas corporales. Las lesiones sospechosas de malignidad a la inspección clínica inicial eran sometidas a continuación al examen dermatoscópico. A diferencia de lo realizado en otros diseños de investigación, en este caso aquellas lesiones de aspecto dermatoscópico no atípico no se biopsiaban, sino que se seguían clínicamente para conocer su evolución. El test de referencia se constituyó por los resultados de las biopsias realizadas, junto con la evolución clínica de las lesiones no biopsiadas en un lapso de cuatro años de seguimiento

Resultados Principales: de 2.542 lesiones examinadas en 133 pacientes, $43(1,6 \%)$ se interpretaron como sospechosas a la inspección clínica. Sin embargo sólo 13 de éstas se biopsiaron luego de la evaluación dermatoscópica. La anatomía patológica confirmo tres melanomas. Se evitaron 30 biopsias. El seguimiento a cuatro años no identificó lesiones malignas en estos pacientes. De esta manera la dermatoscopía produjo un incremento de la

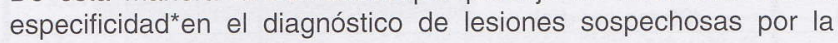

clínica desde $98 \%$ al $99 \%$ mediante el uso de la clínica más la dermatoscopía sin detectarse resultados falsos negativos. Esto se reflejó en un aumento del Valor Predictivo Positivo (VPP)* del 6,9\% con la inspección clínica aislada a un $23 \%$ con la dermatoscopía.

Conclusiones: el agregado de la dermatoscopía al diagnóstico clínico de lesiones sospechosas para melanoma disminuye los diagnósticos falsos positivos, mejora el VPP para el diagnóstico de melanoma y reduce la realización innecesaria de biopsias.

\section{Comentario}

Por los resultados vertidos a partir la de la presente evidencia, estamos en condiciones de afirmar que tanto la inspección clínica como el uso de una herramienta un tanto mas sofisticada, la dermatoscopía, constituyen alternativas eficaces en la detección temprana del melanoma. Así lo demuestra el metanálisis de Bafounta y $\mathrm{col}^{3}$, donde queda además expuesta la superioridad de la dermatoscopía sobre la clínica, y posteriormente el estudio de Carli y $\mathrm{col}^{4}$, que teniendo en cuenta esta base demuestra la aplicabilidad de la dermatoscopía de una manera más pragmática para evitar un problema frecuente en la detección de lesiones melanocíticas malignas, los falsos positivos.

Ambos estudios trabajaron sobre población seleccionada (pacientes derivados o autoderivados), con mayor riesgo y por lo tanto con mayor incidencia de lesiones malignas.

Hay que ser cautos, sin embargo, y tener en cuenta que la performance diagnóstica lograda en estos resultados depende del examen realizado por dermatólogos, muchas veces con alto nivel de especialización. Los reportes de precisión diagnóstica del examen efectuado por médicos de atención primaria son llamativamente menores.

Un punto importante a considerar, que se desprende de la pregunta original, sobre todo para los que hacemos atención primaria, es si debería realizarse rastreo de melanoma. De hecho, de acuerdo a las recomendaciones de la fuerza de tareas preventiva de los Estados Unidos (USPSTF) ${ }^{2}$ el rastreo para melanoma es una recomendación clase I (Indeterminada, evidencia insuficiente), aún para los grupos de alto riesgo. Esta recomendación se fundamenta en la falta de estudios aleatorizados con adecuado seguimiento que evalúen el impacto del screening en la historia natural de la enfermedad (reducción de la morbimortalidad causada por el melanoma). Se ha postulado que el mayor número de diagnósticos tempranos podrían representar en realidad la identificación de un subtipo de neoplasia de comportamiento menos agresivo y esto traducirse en una reducción aparente de la morbimortalidad (lo que se denomina sesgo de anticipación).

Aun luego de resolverse esta importante cuestión también será necesario tener en cuenta estimaciones de costo-efectividad acerca de la eventual incorporación masiva de la dermatoscopía sobre el tradicional examen clínico. Si bien en los países desarrollados la dermatoscopía de epiluminiscencia constituye una tecnología cada vez más incorporada a la práctica dermatológica, en nuestro medio esta herramienta no se encuentra fácilmente disponible, y su costo es varias veces superior al de la consulta dermatológica.

Gabriel Villalón [ Unidad de Medicina Familiar y Preventiva. Hospital Italiano de Buenos Aires. ]

\section{Referencias}

1. Schaw J. Overview of melanoma. In: UpToDate 13.2. Disponible en http://www.uptodate.com

2. Helfand M, Mahon S, Eden K. Screening for skin cancer: a summary of the evidence. American Journal of Preventive Medicine 2001;20(3):47-58.

3. Bafounta ML, Beauchet A, Aegerter P. Is dermoscopy (epiluminescence microscopy) useful for the diagnosis of melanoma?: results of a meta-analysis using techniques adapted to the evaluation of diagnostic test. Archives of dermatology 2001;137(10):1343-1350.

4. Carli P, Mannone F, De Giorgi V. The problem of false-positive diagnosis in melanoma screening: the impact of dermoscopy. Melanoma Research 2003;13:179-182. 